\title{
REMARK ABOUT CHARACTERIZATION OF 2-INNER PRODUCT
}

\author{
Katerina Anevska ${ }^{1}$ and Risto Malčeski ${ }^{2}$
}

\begin{abstract}
Characterization of 2-inner product is focus of interest of many mathematicians. In this paper proofs of two characterizations of 2- inner product, which are actually consequences of the Theorem 1 [15], are given. Also, generalizations of already know Hayashi (see [4], pg. 297) and Zarantonello ([5]) inequalities are fully elaborated.
\end{abstract}

\section{INTRODUCTION}

The concepts of 2-norm and 2-inner product are two-dimensional analogies to the concepts of norm and inner product. S. Gähler ([13]), 1965, gave the term of 2-norm and R. Ehret ([11]), 1969, proved the following:

If $(L,(\cdot, \cdot \mid \cdot))$ is a 2 -pre-Hilbert space, then

$$
\|x, y\|=(x, x \mid y)^{1 / 2} \text {, }
$$

for all $x, y \in L$, defines a 2-norm. So, we get the 2-normed space $(L,\|\cdot, \cdot\|)$ and furthermore for all $\mathrm{x}, \mathrm{y}, \mathrm{z} \in \mathrm{L}$ the following equalities are satisfied:

$$
\begin{aligned}
& (x, y \mid z)=\frac{\|x+y, z\|^{2}-\|x-y, z\|^{2}}{4}, \\
& \|x+y, z\|^{2}+\|x-y, z\|^{2}=2\left(\|x, z\|^{2}+\|y, z\|^{2}\right),
\end{aligned}
$$

The equality (3) is analogue to the parallelogram equality, and it is said to be parallelepiped equality. Moreover, 2-normed space $L$ is 2-pre-Hilbert if and only if the equality (3) is satisfied for all $x, y, z \in L$.

The papers [1]-[3], [6], [12], [14]-[16] consist of many proven characterizations about 2-inner product.

2010 Mathematics Subject Classification. 46C50, 46C15, 46B20

Key words and phrases. 2-norm, 2-inner product, parallelepiped equality 
Theorem 1 ([15]). Let $(L,\|\cdot, \cdot\|)$ be a real 2-normed space. Then, $L$ is a 2-preHilbert space if and only if the following condition is satisfied:

if $n \geq 3, x_{1}, x_{2}, \ldots, x_{n}, z \in L$ and $a_{1}, a_{2}, \ldots, a_{n}$ are real numbers such that $\sum_{i=1}^{n} a_{i}=0$, then

$$
\left\|\sum_{i=1}^{n} a_{i} x_{i}, z\right\|^{2}=-\sum_{1 \leq i<j \leq n} a_{i} a_{j}\left\|x_{i}-x_{j}, z\right\|^{2}
$$

\section{ChaRACTERIZATION OF 2-PRE-HILBERT SPACE}

The characterization of 2-inneer product by applying the Euler-Lagrange type of equality is elaborated in [6] or in other words generalization of Corollary 2.2 [8], is elaborated. The following theorem is one other proof of the above stated generalization.

Theorem 2 ([6]). Let $(L,\|\cdot, \cdot\|)$ be a real 2-normed space. The 2-norm is generated by 2 -inner product if and only if the following equality is satisfied

$$
\frac{\|a x+b y, z\|^{2}}{\gamma}+\frac{\|\beta b x-\alpha a y, z\|^{2}}{\gamma \alpha \beta}=\frac{\|x, z\|^{2}}{\alpha}+\frac{\|y, z\|^{2}}{\beta},
$$

for all $x, y, z \in L$ and for all $a, b \in \mathbf{R}, \alpha, \beta>0, \gamma=\alpha a^{2}+\beta b^{2}$.

Proof. Let $L$ be a real 2-normed space such that for all $x, y, z \in L$ and for all $a, b \in \mathbf{R}, \quad \alpha, \beta>0, \gamma=\alpha a^{2}+\beta b^{2}$ the equality (5) is satisfied. For $\alpha=\beta=a=b=1$, the equality (5) is transformed to an equality which is equivalent to the parallelepiped equality, (3), what actually means that $L$ is 2 pre-Hilbert space in which the 2-inner product is defined as (2) and moreover (1) holds true.

Conversely, let 2-inner product, which determines the 2-norm, exist and let $x, y, z \in L$ and $a, b \in \mathbf{R}, \alpha, \beta>0$ be such that $\gamma=\alpha a^{2}+\beta b^{2}$ is satisfied. For

$$
a_{1}=\frac{a}{\sqrt{\gamma}}, a_{2}=\frac{b}{\sqrt{\gamma}}, a_{3}=-\frac{a+b}{\sqrt{\gamma}}, x_{1}=x, x_{2}=y, x_{3}=0
$$

theorem 1 is transformed as the following

$$
\frac{\|a x+b y, z\|^{2}}{\gamma}=\frac{a(a+b)}{\gamma}\|x, z\|^{2}+\frac{b(a+b)}{\gamma}\|y, z\|^{2}-\frac{a b}{\gamma}\|x-y, z\|^{2} .
$$

Further, for 


$$
a_{1}=\frac{b \sqrt{\beta}}{\sqrt{\alpha \gamma}}, a_{2}=-\frac{a \sqrt{\alpha}}{\sqrt{\beta \gamma}}, a_{3}=-\frac{b \beta-a \alpha}{\sqrt{\alpha \beta \gamma}}, x_{1}=x, x_{2}=y, x_{3}=0
$$

theorem 1 is transformed as the following

$$
\frac{\|\beta b x-\alpha a y, z\|^{2}}{\gamma \alpha \beta}=\frac{b(b \beta-a \alpha)}{\alpha \gamma}\|x, z\|^{2}-\frac{a(b \beta-a \alpha)}{\beta \gamma}\|y, z\|^{2}+\frac{a b}{\gamma}\|x-y, z\|^{2} .
$$

Finally, if we summarize the equalities (6) and (7) and have also on mind that $\gamma=\alpha a^{2}+\beta b^{2}$ we get the following

$$
\begin{aligned}
\frac{\|a x+b y, z\|^{2}}{\gamma}+\frac{\|\beta b x-\alpha a y, z\|^{2}}{\gamma \alpha \beta}= & \left(\frac{a(a+b)}{\gamma}+\frac{b(b \beta-a \alpha)}{\alpha \gamma}\right)\|x, z\|^{2}+ \\
& +\left(\frac{b(a+b)}{\gamma}-\frac{a(b \beta-a \alpha)}{\beta \gamma}\right)\|y, z\|^{2} \\
= & \frac{\alpha a^{2}+\beta b^{2}}{\alpha \gamma}\|x, z\|^{2}+\frac{\alpha a^{2}+\beta b^{2}}{\beta \gamma}\|y, z\|^{2} \\
= & \frac{\|x, z\|^{2}}{\alpha}+\frac{\|y, z\|^{2}}{\beta},
\end{aligned}
$$

i.e. the equality (5) is satisfied.

The following theorem is actually generalization of M. S. Moslehian and J. M. Rassias (Corollary 2.2, [9]) result.

Theorem 3.A real 2- normed space $(L,\|\cdot, \cdot\|)$ is 2- pre-Hilfert space if and only if for each $n \geq 2$ and for all $x_{1}, x_{2}, \ldots, x_{n}, z \in L$ the equality (8) is satisfied

$$
\sum_{a_{i} \in\{-1,1\}}\left\|x_{1}+\sum_{i=2}^{n} a_{i} x_{i}, z\right\|^{2}=\sum_{a_{i} \in\{-1,1\}}\left(\left\|x_{1}, z\right\|+\sum_{i=2}^{n} a_{i}\left\|x_{i}, z\right\|\right)^{2} .
$$

Proof. Let (8) be satisfied for each $n \geq 2$ and for all $x_{1}, x_{2}, \ldots, x_{n}, z \in L$. For $n=2, x_{1}=x$ and $x_{2}=y$ the equality (8) is transformed to the parallelepiped equality (3). That actually means that $L$ is 2 -pre-Hilbert space in which the 2 inner product is defined as (2) and furthermore (1) holds true.

Conversely, let a 2 -normed space $(L,\|\cdot, \cdot\|)$ be a 2-pre-Hilbert space, $n \geq 2$ and $x_{1}, x_{2}, \ldots, x_{n}, z \in L$.

For $a_{n+1}=-\left(1+\sum_{k=2}^{n} a_{i}\right)$ and $x_{n+1}=0$, Theorem 1 is transformed as the following: 


$$
\begin{aligned}
\left\|x_{1}+\sum_{i=2}^{n} a_{i} x_{i}, z\right\|^{2}= & \left\|x_{1}+\sum_{i=2}^{n+1} a_{i} x_{i}, z\right\|^{2} \\
= & \left(1+\sum_{k=2}^{n} a_{k}\right)\left(\left\|x_{1}, z\right\|^{2}+\sum_{i=2}^{n} a_{i}\left\|x_{i}, z\right\|^{2}\right)- \\
& -\sum_{i=2}^{n} a_{i}\left\|x_{1}-x_{i}, z\right\|^{2}-\sum_{2 \leq i<j \leq n} a_{i} a_{j}\left\|x_{i}-x_{j}, z\right\|^{2} \\
= & \sum_{i=1}^{n}\left\|x_{i}, z\right\|^{2}+\left\|x_{1}, z\right\|^{2} \sum_{k=2}^{n} a_{k}+\sum_{k=2}^{n} \sum_{i=2}^{n} a_{k} a_{i}\left\|x_{i}, z\right\|^{2}- \\
& -\sum_{i=2}^{n} a_{i}\left\|x_{1}-x_{i}, z\right\|^{2}-\sum_{2 \leq i<j \leq n} a_{i} a_{j}\left\|x_{i}-x_{j}, z\right\|^{2}
\end{aligned}
$$

and since $a_{i} \in\{-1,1\}$, for $i=2,3, \ldots, n$, we get $2^{n-1}$ equalities of the above type. By summarizing the such obtained equalities, we get the following.

$$
\begin{aligned}
\sum_{a_{i} \in\{-1,1\}}\left\|x_{1}+\sum_{i=2}^{n} a_{i} x_{i}, z\right\|^{2} & =2^{n-1} \sum_{i=1}^{n}\left\|x_{i}, z\right\|^{2}+\sum_{a_{k} \in\{-1,1\}} \sum_{k=2}^{n} a_{k}\left\|x_{1}, z\right\|^{2}+ \\
& +\sum_{a_{k}, a_{i} \in\{-1,1\}} \sum_{\substack{k=2 \\
i}}^{n} \sum_{\substack{i=k \\
i \neq k}}^{n} a_{k} a_{i}\left\|x_{i}, z\right\|^{2}- \\
& -\sum_{a_{k}, a_{i} \in\{-1,1\}} \sum_{i=2}^{n} a_{i}\left\|x_{1}-x_{i}, z\right\|^{2}- \\
& -\sum_{a_{k}, a_{i} \in\{-1,1\} 2 \leq i<j \leq n} a_{i} a_{j}\left\|x_{i}-x_{j}, z\right\|^{2} \\
= & 2^{n-1} \sum_{i=1}^{n}\left\|x_{i}, z\right\|^{2} \\
= & \sum_{a_{i} \in\{-1,1\}}\left(\left\|x_{1}, z\right\|+\sum_{i=2}^{n} a_{i}\left\|x_{i}, z\right\|\right)^{2},
\end{aligned}
$$

i.e. the equality (8) holds true.

\section{GENERALIZATION OF HAYASHI AND ZARANTONELlo INEQUALITIES}

The following theorems, are actually generalization of two already known equalities, obtained by using theorem 1. Thus, we will firstly give a generalization of Hayashi (see [4], pg. 297) inequality for complex numbers. 
Theorem 4. Let $(L,\|\cdot, \cdot\|)$ be a real 2-normed space. Then

$$
\sum_{\text {cyclic }}\left\|x-x_{1}, z\right\| \cdot\left\|x-x_{2}, z\right\| \cdot\left\|x_{1}-x_{2}, z\right\| \geq\left\|x_{1}-x_{2}, z\right\| \cdot\left\|x_{2}-x_{3}, z\right\| \cdot\left\|x_{3}-x_{1}, z\right\|
$$

for all $x, x_{1}, x_{2}, x_{3}, z \in L$. The inequality is transformed to an equality, if at least one of the sets $\left\{x-x_{1}, z\right\},\left\{x-x_{2}, z\right\},\left\{x-x_{3}, z\right\}$ is linearly dependent or more over if the set

$$
\left\{\frac{\left\|x_{2}-x_{3}, z\right\|}{\left\|x-x_{1}, z\right\|}\left(x-x_{1}\right)+\frac{\left\|x_{3}-x_{1}, z\right\|}{\left\|x-x_{2}, z\right\|}\left(x-x_{2}\right)+\frac{\left\|x_{1}-x_{2}, z\right\|}{\left\|x-x_{3}, z\right\|}\left(x-x_{3}\right), z\right\}
$$

is linearly dependent.

Proof. Let at least one of the sets $\left\{x-x_{1}, z\right\},\left\{x-x_{2}, z\right\},\left\{x-x_{3}, z\right\}$ be linearly dependent. With no loose of the generality, let $\left\{x-x_{1}, z\right\}$ be such the set, i.e. $x=x_{1}+\alpha z$. Then, the properties of 2-norm imply the following

$$
\begin{gathered}
\sum_{\text {cyclic }}\left\|x-x_{1}, z\right\| \cdot\left\|x-x_{2}, z\right\| \cdot\left\|x_{1}-x_{2}, z\right\|=\left\|x-x_{2}, z\right\| \cdot\left\|x-x_{3}, z\right\| \cdot\left\|x_{2}-x_{3}, z\right\| \\
=\left\|x_{1}+\alpha z-x_{2}, z\right\| \cdot\left\|x_{1}+\alpha z-x_{3}, z\right\| \cdot\left\|x_{2}-x_{3}, z\right\| \\
=\left\|x_{1}-x_{2}, z\right\| \cdot\left\|x_{2}-x_{3}, z\right\| \cdot\left\|x_{3}-x_{1}, z\right\|,
\end{gathered}
$$

The above means that (9) is an equality.

Let's suppose that the sets $\left\{x-x_{1}, z\right\},\left\{x-x_{2}, z\right\},\left\{x-x_{3}, z\right\}$ are linearly independent. For $a_{4}=-\sum_{i=1}^{3} a_{i}$ and $x_{4}=x$ in Theorem 1, we get that for all $x, x_{1}, x_{2}, x_{3}, z \in L$ and for all $a_{1}, a_{2}, a_{3} \in \mathbf{R}$ the equality

$$
\left\|\sum_{i=1}^{3} a_{i} x_{i}-x \sum_{i=1}^{3} a_{i}, z\right\|^{2}=\left(\sum_{i=1}^{3} a_{i}\right) \cdot\left(\sum_{i=1}^{3} a_{i}\left\|x-x_{i}, z\right\|\right)-\sum_{1 \leq i<j \leq 3} a_{i} a_{j}\left\|x_{i}-x_{j}, z\right\|^{2}
$$

holds true.

The right side of the above equality is nonnegative. Therefore, for all $x, x_{1}, x_{2}, x_{3}, z \in L$ and for all $a_{1}, a_{2}, a_{3} \in \mathbf{R}$ the inequality (10) holds true

$$
\left(\sum_{i=1}^{3} a_{i}\right) \cdot\left(\sum_{i=1}^{3} a_{i}\left\|x-x_{i}, z\right\|\right) \geq \sum_{1 \leq i<j \leq 3} a_{i} a_{j}\left\|x_{i}-x_{j}, z\right\|^{2} .
$$

For

$$
a_{1}=\frac{\left\|x_{2}-x_{3}, z\right\|}{\left\|x-x_{1}, z\right\|}, a_{2}=\frac{\left\|x_{3}-x_{1}, z\right\|}{\left\|x-x_{2}, z\right\|}, a_{3}=\frac{\left\|x_{1}-x_{2}, z\right\|}{\left\|x-x_{3}, z\right\|}
$$

the inequality (10) is transformed as the followings

$$
\sum_{i \neq j \neq k \neq i} \frac{\left\|x_{i}-x_{j}, z\right\|}{\left\|x-x_{k}, z\right\|} \sum_{i \neq j \neq k \neq i}\left\|x_{i}-x_{j}, z\right\| \cdot\left\|x-x_{k}, z\right\| \geq \sum_{i \neq j \neq k \neq i} \frac{\left\|x_{j}-x_{k}, z\right\|}{\left\|x-x_{i}, z\right\|} \frac{\left\|x_{k}-x_{i}, z\right\|}{\left\|x-x_{j}, z\right\|}\left\|x_{i}-x_{j}, z\right\|^{2}
$$




$$
\begin{gathered}
\sum_{i \neq j \neq k \neq i} \frac{\left\|x_{i}-x_{j}, z\right\|}{\left\|x-x_{k}, z\right\|} \sum_{i \neq j \neq k \neq i}\left\|x_{i}-x_{j}, z\right\| \cdot\left\|x-x_{k}, z\right\| \geq \\
\quad \geq \frac{\left\|x_{1}-x_{2}, z\right\| \cdot\left\|x_{2}-x_{3}, z\right\| \cdot\left\|x_{3}-x_{1}, z\right\|}{\left\|x-x_{1}, z\right\| \cdot\left\|x-x_{2}, z\right\| \cdot\left\|x-x_{3}, z\right\|} \sum_{i \neq j \neq k \neq i}\left\|x_{i}-x_{j}, z\right\| \cdot\left\|x-x_{k}, z\right\| \\
\sum_{i \neq j \neq k \neq i} \frac{\left\|x_{i}-x_{j}, z\right\|}{\left\|x-x_{k}, z\right\|} \geq \frac{\left\|x_{1}-x_{2}, z\right\| \cdot\left\|x_{2}-x_{3}, z\right\| \cdot\left\|x_{3}-x_{1}, z\right\|}{\left\|x-x_{1}, z\right\| \cdot\left\|x-x_{2}, z\right\| \cdot\left\|x-x_{3}, z\right\|} .
\end{gathered}
$$

Clearly, the last inequality is equivalent to the inequality (9). The proof implies that the inequality (9) might be transformed to an equality if (10) is an equality, i.e. if the set $\left\{\sum_{i=1}^{3} a_{i} x_{i}-x \sum_{i=1}^{3} a_{i}, z\right\}$ is linearly dependent, that is if the set

$$
\left\{\frac{\left\|x_{2}-x_{3}, z\right\|}{\left\|x-x_{1}, z\right\|}\left(x-x_{1}\right)+\frac{\left\|x_{3}-x_{1}, z\right\|}{\left\|x-x_{2}, z\right\|}\left(x-x_{2}\right)+\frac{\left\|x_{1}-x_{2}, z\right\|}{\left\|x-x_{3}, z\right\|}\left(x-x_{3}\right), z\right\}
$$

is linearly dependent.

On the end of our considerations we will generalize the Zarantonello ([5]), inequality, i.e. we will prove the following theorem.

Theorem 5. Let $L$ be a real 2-pre-Hilbert space and $f: L \rightarrow L$ be a function such that

$$
\|f(x)-f(y), z\| \leq\|x-y, z\|
$$

holds true, for all $x, y, x \in L$, Then for all $a_{1}, a_{2}, \ldots, a_{n} \geq 0$, such that $\sum_{i=1}^{n} a_{i}=1$ and for all $y_{1}, y_{2}, \ldots, y_{n}, z \in L$

$$
\left\|\sum_{i=1}^{n} a_{i} f\left(y_{i}\right)-f\left(\sum_{k=1}^{n} a_{k} y_{k}\right), z\right\|^{2} \leq \sum_{1 \leq i<k \leq n} a_{i} a_{k}\left(\left\|y_{i}-y_{k}, z\right\|^{2}-\left\|f\left(y_{i}\right)-f\left(y_{k}\right), z\right\|^{2}\right)
$$

holds true.

Proof. For

$$
x_{i}=f\left(y_{i}\right), i=1,2, . ., . n, x_{n+1}=f\left(\sum_{i=1}^{n} a_{i} y_{i}\right)
$$

and $a_{n+1}=-1$, in Theorem 1 and then by using the inequality (11) and the properties of 2-norm, we get that for all $a_{1}, a_{2}, \ldots, a_{n} \geq 0$ such that $\sum_{i=1}^{n} a_{i}=1$ and for all $y_{1}, y_{2}, \ldots, y_{n}, z \in L$, the following holds true 


$$
\begin{aligned}
\| \sum_{i=1}^{n} a_{i} f\left(y_{i}\right) & -f\left(\sum_{k=1}^{n} a_{k} y_{k}\right), z \|^{2}= \\
& =\sum_{i=1}^{n} a_{i}\left\|f\left(y_{i}\right)-f\left(\sum_{k=1}^{n} a_{k} y_{k}\right), z\right\|^{2}-\sum_{1 \leq i<k \leq n} a_{i} a_{k}\left\|f\left(y_{i}\right)-f\left(y_{k}\right), z\right\|^{2} \\
& \leq \sum_{i=1}^{n} a_{i}\left\|y_{i}-\sum_{k=1}^{n} a_{k} y_{k}, z\right\|^{2}-\sum_{1 \leq i<k \leq n} a_{i} a_{k}\left\|f\left(y_{i}\right)-f\left(y_{k}\right), z\right\|^{2} \\
& =\sum_{i=1}^{n} a_{i}\left\|\sum_{k=1}^{n} a_{k} y_{i}-\sum_{k=1}^{n} a_{k} y_{k}, z\right\|^{2}-\sum_{1 \leq i<k \leq n} a_{i} a_{k}\left\|f\left(y_{i}\right)-f\left(y_{k}\right), z\right\|^{2} \\
& =\sum_{i=1}^{n} a_{i}\left\|\sum_{k=1}^{n} a_{k}\left(y_{i}-y_{k}\right), z\right\|^{2}-\sum_{1 \leq i<k \leq n} a_{i} a_{k}\left\|f\left(y_{i}\right)-f\left(y_{k}\right), z\right\|^{2} .
\end{aligned}
$$

On the other hand, for $x_{k}=y_{i}-y_{k}, k=1,2, \ldots, n, x_{n+1}=0$ and $a_{n+1}=-1$ in Theorem 1 and also by using that $a_{i} \geq 0$, for $i=1,2, \ldots, n$, we get that

$$
\begin{aligned}
\left\|\sum_{k=1}^{n} a_{k}\left(y_{i}-y_{k}\right), z\right\|^{2} & =\sum_{k=1}^{n} a_{k}\left\|y_{i}-y_{k}, z\right\|^{2}-\sum_{1 \leq i<j \leq n} a_{i} a_{j}\left\|y_{i}-y_{j}, z\right\|^{2} \\
\leq & \sum_{k=1}^{n} a_{k}\left\|y_{i}-y_{k}, z\right\|^{2} .
\end{aligned}
$$

Finally, the last two inequalities imply the inequality (12).

\section{CONFLICT OF INTEREST}

No conflict of interest was declared by the authors.

\section{Author's Contributions}

All authors contributed equally and significantly to writing this paper. All authors read and approved the final manuscript.

\section{References}

[1] C. Diminnie, A. White, 2-Inner Product Spaces and Gâteaux partial derivatives, Comment Math. Univ. Carolinae 16(1) (1975), 115-119

[2] C. Diminnie, A. White, A characterizatin of 2-inner product spaces, Math. Japon. No. 27 (1982), 357-377. 
[3] C. Diminnie, S. Gähler, A. White, 2-Inner Product Spaces, Demonstratio Mathematica, Vol. VI (1973), 525-536

[4] D. S. Mitrinović, J. E. Pečarić, V. Volenec, Recent Advances in Geometric Inequalities, Kluwer Academic Publisher, 1989

[5] E. H. Zarantonello, Projections on convex sets in Hilbert spaces and spectral theory, Contributions to Nonlinear Functional Analysis, Acad. Press., New York, 1971, 237-424

[6] K. Anevska, R. Malčeski, Characterization of 2-inner product using EulerLagrange type of equality, International Journal of Science and Research (IJSR), ISSN 2319-7064, Vol. 3 Issue 6 (2014), 1220-1222.

[7] M. Fréchet, Sur la definition axiomatique d'une classe d'espaces vectoriels distanciés applicable vectorillement sur l'espace de Hilbert, Ann. of Math. Vol. 36 (3) (1935), 705-718.

[8] M. S. Moslehian, J. M. Rassias, A characterization of inner product spaces concerning an Euler-Lagrange identity, Commun. Math. Anal. Vol. 8, no. 2 (2010), 16-21

[9] M. S. Moslehian, J. M. Rassias, A characterization of inner product spaces, Kochi. J. Math., Vol. 6 (2011), 101-111

[10] P. Jordan, J. von Neumann, On inner products in linear, metric spaces, Ann. of Math. Vol. 36 (3) (1935), 719-723.

[11] R. Ehret, Linear 2-Normed Spaces, Doctoral Diss., Saint Louis Univ., 1969

[12] R. Malčeski, K. Anevska, Characterization of 2-inner product by strictly convex 2-norm of modul c, International Journal of Mathematical Analysis, Vol. 8, no. 33 (2014), 1647-1652

[13] S. Gähler, Lineare 2-normierte Räume, Math. Nachr. 28 (1965), 1-42

[14] S. Malčeski, A. Malčeski, K. Anevska, R. Malčeski, Another characterizations of 2-pre-Hilbert space, IJSIMR, e-ISSN 2347-3142, pISSN 2346-304X, Vol. 3, Issue 2 (2015), pp. 45-54.

[15] S. Malčeski, K. Anevska, R. Malčeski, New characterization of 2-preHilbert Space, Matematički bilten, Vol. 38(XLIV) no. 1 (2014), 31-38

[16] Y. J. Cho, S. S. Kim, Gâteaux derivatives and 2-Inner Product Spaces, Glasnik Math., Vol. 27 (47) (1992), 271-282

[17] D. S. Marinescu, M. Monea, M. Opimcariu, M. Stroe, Some Equivalent Characterizations of Inner Product Spaces and Their Consequences, Filomat 29 (7) (2015), 1587-1599

\footnotetext{
${ }^{1)}$ Faculty of informatics, FON University, Skopje, Macedonia E-mail address: anevskak@gmail.com

${ }^{2)}$ Faculty of informatics, FON University, Skopje, Macedonia E-mail address: risto.malceski@gmail.com
} 